\section{Transformation, Log-}

R.-D. Hilgers ${ }^{1}$, N. Heussen ${ }^{1}$ und S. Stanzel ${ }^{2}$

${ }^{1}$ Institut für Medizinische Statistik, Universitätsklinikum der RWTH Aachen, Aachen, Deutschland

${ }^{2}$ DKFZ Heidelberg, Heidelberg, Deutschland

Synonym(e) Logarithmische Transformation

Englischer Begriff log transformation; logarithmic transformation
Definition Die Log-Transformation ordnet jedem ursprünglichen Wert als transformierten Wert den entsprechenden natürlichen Logarithmus dieses Werts zu.

Beschreibung Die Log-Transformation wird vor allem zur - Transformation von Daten aus einer $>$ Log-Normalverteilung in solche aus einer $>$ Normalverteilung verwandt. Diese Transformation sollte nur dann angewendet werden, wenn die beobachteten Daten Werte größer als Null annehmen.

\section{Literatur}

Hartung J, Elpelt B, Klösener KH (1995) Statistik - Lehr- und Handbuch der angewandten Statistik. Oldenbourg Verlag, München 